\title{
UTILISATION EN FROMAGERIE DE GRUYÈRE DE SUSPENSIONS CONCENTRÉES ET CONGELÉES DE BACTÉRIES LACTIQUES THERIMOPHILES (1)
}

\author{
par \\ P. ROUSSEAUX, L. VASSAL, E. VALLES, J. AUCLAIR \\ et G. MOCQUOT \\ Station expérimentale laitière, Poligny, Jura \\ Station centrale de Recherches laitières et de technologie \\ des Produits animaux, I.N.R.A., Jouy-en-Josas, Yvelines
}

\section{Introduction}

Dans un article précédent [1] nous avons montré l'intérêt que pouvait présenter pour l'industrie fromagère l'utilisation de suspensions concentrées de bactéries lactiques, conservées à l'état congelé, pour l'ensemencement du lait de fromagerie, à la place des levains sur lait traditionnels. De telles suspensions permettent d'ensemencer directement le lait de fabrication en évitant au fromager la préparation des levains et toutes les difficultés qui souvent en découlent.

Dans le présent travail, nous donnons les résultats d'expériences portant sur la fabrication de Gruyère de Comté à l'aide de suspensions concentrées de bactéries thermophiles. La technique de préparation de ces suspensions sera décrite dans une autre publication [2]. Les expériences qui vont être décrites ont été réalisées, d'une part à la Laiterie expérimentale de Jouy-en-Josas, d'autre part dans quatre fromageries du Jura.

\section{Méthodes}

A) Suspensions.

L'ensemble des essais a été réalisé à l'aide de suspensions concentrées de deux souches bactériennes de la collection du Centre national de Recherches zootechniques: Str. thermophilus CNRZ 302 et L. helveticus CNRZ 303.

Les suspensions concentrées de ces germes (streptocoques et lactobacilles) contenaient généralement entre $2.10^{10}$ et $5.10^{10}$ bac-

(1) Ce travail a bénéficié d'une subvention de la Délégation générale à la Recherche scientifique et technique (contrat 65-FR-169). 
téries par gramme, c'est-à-dire 10 à 100 fois plus que les levains sur lait habituellement utilisés en fromagerie. Elles étaient conditionnées soit dans des tubes d'aluminium, soit dans des flacons de verre, à raison de $5 \mathrm{~g}$ pour les streptocoques et de $25 \mathrm{~g}$ pour les lactobacilles. Les tubes et flacons étaient congelés immédiatement après leur préparation à $-30^{\circ} \mathrm{C}$ et conservés ensuite à cette même température.

\section{B) Essais préliminaires en fromagerie. 1}

Dans ces premiers essais, nous nous sommes limités à étudier l'influence de l'utilisation de suspensions concentrées de bactéries thermophiles sur l'acidification du fromage sous presse, la teneur en extrait sec du fromage de 24 heures et le rendement en fromage frais obtenu pour 100 litres de lait.

Sept fabrications de Gruyère ont ainsi été effectuées à la laiterie expérimentale de Jouy-en-Josas en utilisant comparativement des levains préparés sur lait stérilisé et des suspensions bactériennes concentrées congelées.

A chaque essai on fabriquait deux fromages, à partir du même lait provenant d'un des troupeaux du Centre national de Recherches zootechniques. Le lait, préalablement ajusté à un taux de graisse déterminé, était réparti dans deux cuves, et le volume de lait dans chaque cuve était mesuré avec une précision de $\pm 1 \mathrm{p}$. 100. Pour l'un des fromages on utilisait comme levain deux cultures de 16 heures sur lait stérilisé de Str. thermophilus 302 et de $L$. helveticus 303, que l'on ajoutait au lait de chaudière à raison de 0,2 p. 100 pour chacune des deux cultures.

L'autre fromage était fait à l'aide de suspensions concentrées congelées des mêmes souches, qui étaient ajoutées au lait de chaudière à raison de 0,001 p. 100 pour Str. thermophilus et de 0,01 p. 100 pour L. helveticus. Pratiquement on ajoutait, à une cuve contenant 500 à 600 litres de lait, un flacon de $5 \mathrm{~g}$ de suspension de Str. thermophilus et deux flacons de $25 \mathrm{~g}$ de L. helveticus. Les flacons, pris au congélateur à $-30^{\circ} \mathrm{C}$, étaient réchauffés dans un bain-marie à $40^{\circ} \mathrm{C}$ juste le temps suffisant pour que les suspensions congelées puissent se détacher facilement des flacons pour être versées directement dans le lait.

Après la coagulation du lait, obtenue par l'emploi de présure liquide de force 10000 , le travail en cuve (découpage du caillé, chauffage à $54^{\circ} \mathrm{C}$ et brassage) était conduit de la même façon pour les deux fromages en respectant les normes habituelles de la fabrication de Gruyère. A la fin du travail en cuve, la température du contenu de la cuve était amenée à $49^{\circ} \mathrm{C}$ par ruissellement d'eau froide sur la paroi extérieure de la cuve.

Les fromages étaient ensuite moulés sous sérum dans des moules métalliques à l'aide d'un dispositif construit par les Etablis- 
sements Chalon et Megard. Ce dispositif présente, entre autres, l'avantage d'éliminer la perte de caillé connue des fromagers sous le nom de "recherchon ". Après 6 heures de pressage sous des presses pneumatiques du même constructeur, les fromages étaient retournés et pressés encore pendant 16 à 18 heures avant d'être démoulés. La température de la salle de pressage était maintenue constante à $22^{\circ} \mathrm{C}$.

L'acidification du fromage sous presse était contrôlée par la détermination $\mathrm{du} p \mathrm{H}$ du fromage au moment du retournement (après 6 heures de pressage) et au démoulage.

La teneur du fromage en matière sèche était déterminée 24 heures après l'emprésurage par dessiccation à l'étuve à $99-100^{\circ} \mathrm{C}$ d'un échantillon moyen prélevé sur toute la hauteur du fromage, au centre de la meule.

Le poids des fromages sortant de presse était déterminé avec une précision de $\pm 100 \mathrm{~g}$.

C) Utilisation de suspensions concentrées congelées en fromagerie de Gruyère.

Des suspensions concentrées des souches de Str. thermophilus 302 et $L$. helveticus 303 ont été utilisées de décembre 1966 à mars 1967 dans quatre fromageries de la région du Jura.

Les suspensions préparées au Laboratoire de Jouy-en-Josas étaient envoyées alternativement aux quatre fromageries, qui les utilisaient chaque jour, pendant une période de 7 jours consécutifs, pour ensemencer une cuve à fromage. Chaque fromagerie recevait ainsi toutes les 4 semaines un nouveau lot de suspensions concentrées.

a) Transport et conservation des suspensions dans les fromageries.

Les suspensions congelées, conditionnées comme précédemment par doses de $5 \mathrm{~g}$ pour les streptocoques et de $25 \mathrm{~g}$ pour les lactobacilles, étaient transportées aux fromageries dans des boîtes isothermes contenant de la glace carbonique. A l'arrivée à la fromagerie, les flacons étaient conservés pendant les 7 jours d'utilisation dans la glace carbonique.

b) Fabrication et affinage du fromage.

Chaque fromagerie fabriquait chaque jour, pendant les périodes d'essai de 7 jours, un fromage dit "fromage d'essai " à l'aide des suspensions concentrées de streptocoques et de lactobacilles et un autre fromage, dit "fromage témoin », à l'aide du levain traditionnel ("présure aux caillettes").

Pour les fromages d'essai, les doses de suspension utilisées pour l'ensemencement du lait de chaudière étaient de $5 \mathrm{~g}$ de suspension de streptocoques et de $50 \mathrm{~g}$ de suspension de lactobacilles pour 500 à 600 litres de lait. L'emprésurage était fait à l'aide de présure liquide du commerce de force 10000. 
Dans le cas des fromages témoins, l'emprésurage (et l'ensemencement) du lait était réalisé par addition de 1,5 à 2 litres de "présure aux caillettes" pour 500 à 600 litres de lait.

Dans chaque fromagerie, la technique de fabrication était celle utilisée habituellement par le fromager. Cependant, dans tous les eas, le lait du soir destiné à la fabrication des fromages d'essai, était ensemencé à des doses variant entre 0,007 et 0,01 p. 100 à l'aide d'un levain sur lait de streptocoques lactiques mésophiles fourni par la Station expérimentale laitière de Poligny (mélange de 4 souches appartenant aux espèces Str. lactis, Str. cremoris, Str. diacetilactis).

Les fromages (essais et témoins) des quatre fromageries étaient affinés dans les mêmes conditions : séjour de 15 à 45 jours dans les caves des fromageries ( 12 à $14^{\circ} \mathrm{C}$ ), suivi d'un séjour de 1 mois $1 / 2$ à 2 mois $1 / 2$ en cave d'affinage à $15-17^{\circ} \mathrm{C}$. Ils étaient ensuite remis en cave froide (11 à $\left.14^{\circ} \mathrm{C}\right)$. La durée totale de l'affinage a varié suivant les fromages entre 4 mois et 6 mois $1 / 2$.

c) Examen des fromages après affinage.

Après affinage, sur les 84 fromages d'essai fabriqués dans les quatre fromageries au cours de 3 périodes d'essai de 7 jours, 70 ont été coupés et examinés par un jury de 3 à 5 experts.

L' "ouverture ) (c'est-à-dire le nombre et la dimension des "yeux" sur une coupe du fromage selon un diamètre), le goût et la pâte étaient notés sur 10 points. La note totale du fromage était obtenue par l'addition des points affectés des coefficients suivants :

$\begin{array}{ll}\text { Ouverture : } & 5 \\ \text { Goût : } & 6 \\ \text { Pâte : } & 4\end{array}$

Le maximum de points pouvant être attribués à un fromage était done de 150 .

Parallèlement, 70 fromages témoins, coupés et examinés en même temps que les fromages d'essai correspondants, ont été notés de la même façon.

\section{Résultats}

\section{A) Essais préliminaires.}

Les résultats de ces essais figurent au tableau I. Après 6 heures de pressage, les valeurs de $p \mathrm{H}$ mesurées sur les fromages préparés à partir de suspensions sont souvent plus faibles que celles du fromage témoin préparé avec le levain sur lait. Les valeurs de $p H$ obtenues après 24 heures sont sensiblement les mêmes pour les deux séries de fromages. Les suspensions concentrées se comportent donc, aux doses utilisées, comme des levains très actifs, capables d'acidifier rapidement le fromage. 


\section{TABLEAU I}

FABRICATION EXPÉRIMENTALE DE FROMAGES DE GRUYÈRE A L'AIDE DE SUSPENSIONS CONCENTRÉS DE BACTÉRIES THERMOPHILES. MESURE DE L'ACIDIFICATION SOUS PRESSE ET DE L'ÉGOUTTAGE

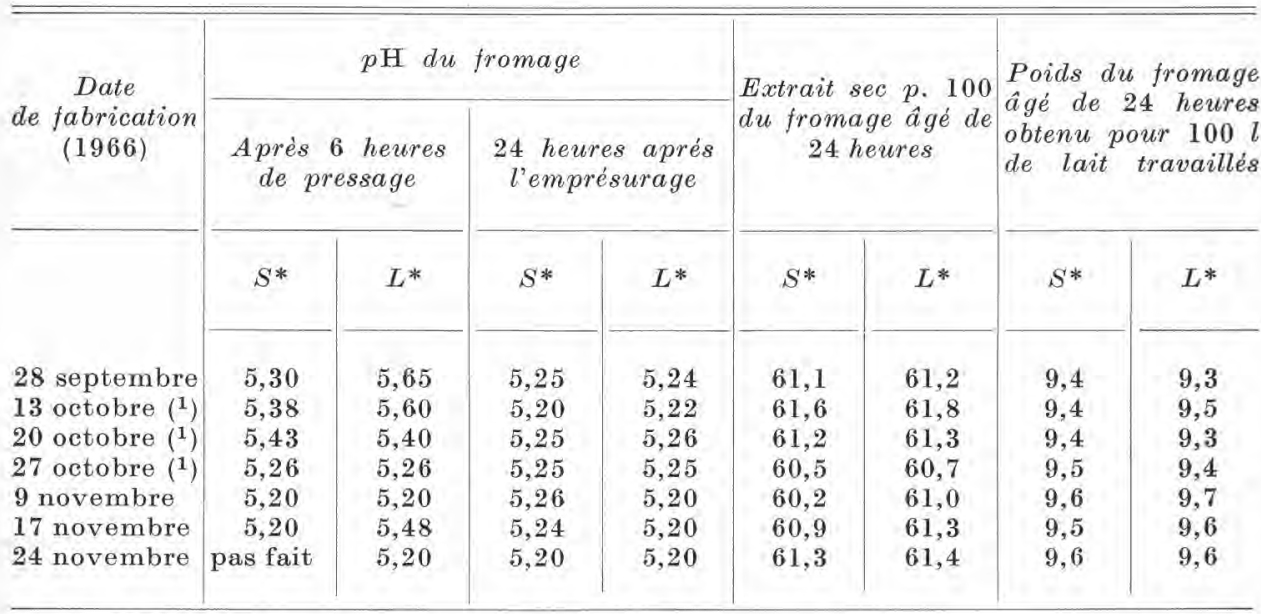

*L : Levains sur lait ; $\mathbf{S}$ : suspensions concentrées congelées.

(1) Au cours de ces fabrications, seul Str. thermophilus a été apporté sous forme de suspensions. L. helveticus était inoculé dans le lait de fabrication sous forme de culture sur lait.

La reproductibilité du phénomène d'acidification est également meilleure d'un essai à l'autre avec les suspensions qu'avec les levains sur lait. Ainsi, sur les 6 essais pour lesquels le $p H$ a été mesuré après 6 heures de pressage, les valeurs obtenues avec les suspensions sont comprises entre 5,20 et 5,43 , soit dans un intervalle de 0,23 unité $p \mathbf{H}$, alors qu'avec les levains sur lait elles sont comprises entre 5,20 et 5,65, soit dans un intervalle de 0,45 unité $p \mathrm{H}$.

Vingt-quatre heures après l'emprésurage, les teneurs en matière sèche sont pratiquement identiques pour les deux types de fromage.

Le poids de fromage obtenu à partir de 100 litres de lait est également le même pour les deux sortes de levains, à la précision des mesures près. Au total, pour ces 7 essais, on a obtenu avec les suspensions concentrées $352,8 \mathrm{~kg}$ de fromage pour 3715 litres de lait, et avec les levains sur lait $352,2 \mathrm{~kg}$ de fromage pour 3710 litres du même lait.

B) Expérimentation en fromagerie de Gruyère.

Les tableaux II et III résument les résultats obtenus pour l'ensemble des fromages fabriqués entre décembre 1966 et mars 1967, dans les quatre fromageries en expérience. La comparaison 
des notes obtenues par les fromages d'essai et les fromages témoins, pour les 70 paires de fromages examinées, montre que dans 46 cas le fromage d'essai a obtenu une note supérieure au fromage témoin correspondant. Le fromage témoin a obtenu 19 fois une note supérieure au fromage d'essai correspondant. Pour 5 paires la note attribuée au fromage d'essai et au fromage témoin était la même. Le tableau II donne la répartition de ces appréciations pour les 4 fromageries.

\section{TABLEAU II}

UTILISATION DE SUSPENSIONS CONCENTRÉES ET CONGELÉES DE BACTÉRIES THERMOPHILES EN FROMAGERIE DE GRUYĖE. COMPARAISON DES NOTES DES FROMAGES GROUPÉS PAR PAIRES

\begin{tabular}{c|c|c|c|c|c}
\hline \hline & $\begin{array}{c}\text { Froma- } \\
\text { gerie } \\
\text { A }\end{array}$ & $\begin{array}{c}\text { Froma- } \\
\text { gerie } \\
\text { B }\end{array}$ & $\begin{array}{c}\text { Froma- } \\
\text { gerie } \\
\text { C }\end{array}$ & $\begin{array}{c}\text { Froma- } \\
\text { gerie } \\
\text { D }\end{array}$ & Total \\
\hline $\begin{array}{c}\text { Nombre de paires de fromages } \\
\text { fabriquées .............. }\end{array}$ & 21 & 21 & 21 & 21 & 84 \\
$\begin{array}{c}\text { Nombre de paires de fromages } \\
\text { examinées ............... }\end{array}$ & 13 & 18 & 18 & 21 & 70 \\
$\begin{array}{c}\text { Nombre de fois où les fromages } \\
\text { d'essai ont obtenu une note } \\
\text { supérieure à celle du témoin ... }\end{array}$ & 9 & 10 & 15 & 12 & 46 \\
$\begin{array}{c}\text { Nombre de fois où les fromages } \\
\text { d'essai ont obtenu une note } \\
\text { inférieure à celle du témoin ... }\end{array}$ & 2 & 6 & 2 & 9 & 19 \\
$\begin{array}{c}\text { Nombre de fois où les fromages } \\
\text { d'essai ont obtenu une note } \\
\text { égale à celle du témoin ....... }\end{array}$ & 2 & 2 & 1 & 0 & 5 \\
\hline \hline
\end{tabular}

Les moyennes des notes obtenues par les fromages d'essai et par les fromages témoins figurent au tableau III. Pour alléger la présentation, ce tableau ne porte pas toutes les notes des fromages mais seulement les moyennes obtenues par les fromages fabriqués pendant les périodes de 7 jours.

La note moyenne obtenue pour l'ensemble des fromages notés (essais et témoins réunis) est de 95,5 sur 150 points. Dans l'ensemble la qualité des fromages fabriqués était donc satisfaisante. En fait, la qualité moyenne des fromages variait suivant les fromageries. Les fromageries $A$ et $B$ peuvent être considérées comme très bonnes; elles donnaient en général des produits dont la qualité était nettement supérieure à celle des fromages des fromageries $\mathrm{C}$ et $\mathrm{D}$ (notes moyennes 108,5 et 105,5 contre 85,0 et 82,5 ).

Les moyennes des notes obtenues par les fromages d'essai et les fromages témoins montrent en général un avantage en faveur des fromages d'essai. 
TABLEAU III

UTILISATION DE SUSPENSIONS CONCENTRÉES ET CONGELÉES DE BACTÉRIES THERMOPHILES EN FROMAGERIE DE GRUY ÈRE. MOYENNES DES NOTES OBTENUES PAR LES FROMAGES ESSAIS ET TÉMOINS

\begin{tabular}{|c|c|c|c|c|c|c|c|c|c|c|}
\hline \multirow{3}{*}{ Période des essais } & \multicolumn{8}{|c|}{ Classement par fromagerie } & \multirow{2}{*}{\multicolumn{2}{|c|}{$\begin{array}{c}\text { Moyenne } \\
\text { des } 4 \text { fromageries }\end{array}$}} \\
\hline & \multicolumn{2}{|c|}{ Fromagerie $A$} & \multicolumn{2}{|c|}{ Fromagerie $B$} & \multicolumn{2}{|c|}{ Fromagerie $C$} & \multicolumn{2}{|c|}{ Fromagerie $D$} & & \\
\hline & Essais & Témoins & Essais & Témoins & Essais & Témoins & Essais & Témoins & Essais & Témoins \\
\hline $\begin{array}{c}\text { Décembre 1966- } \\
\text { janvier } 1967 \ldots\end{array}$ & 113,7 & 109,5 & 125,5 & 120,3 & 115,1 & 80,6 & 101,9 & 84,1 & 114,1 & 98,6 \\
\hline Janvier-février $1967 \ldots$ & 116,4 & 104,7 & 80,9 & 96,9 & 76,1 & 62,2 & 84,1 & 69,1 & 89,4 & 83,2 \\
\hline Février-mars $1967 \ldots$ & 107,5 & 95,4 & 102,9 & 92,1 & 86,4 & 84,2 & 76,6 & 78,9 & 93,4 & 78,7 \\
\hline Moyenne des 3 périodes & 113,3 & 104,4 & 106,8 & 104,1 & 93,1 & 76,9 & 87,5 & 77,4 & 100,2 & 90,7 \\
\hline Note moyenne....... & \multicolumn{2}{|c|}{108,8} & \multicolumn{2}{|c|}{105,5} & \multicolumn{2}{|c|}{85,0} & \multicolumn{2}{|c|}{82,5} & \multicolumn{2}{|c|}{95,5} \\
\hline
\end{tabular}


A $2913 \mathrm{E}$

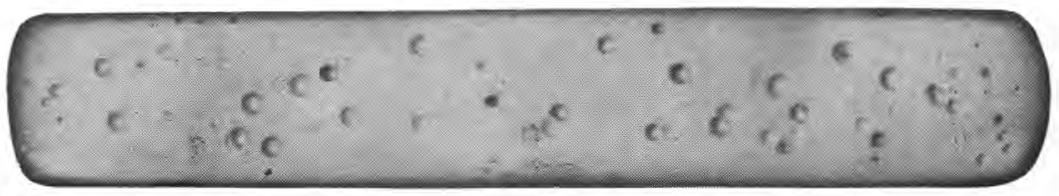

\section{A $2912 \mathrm{~T}$}

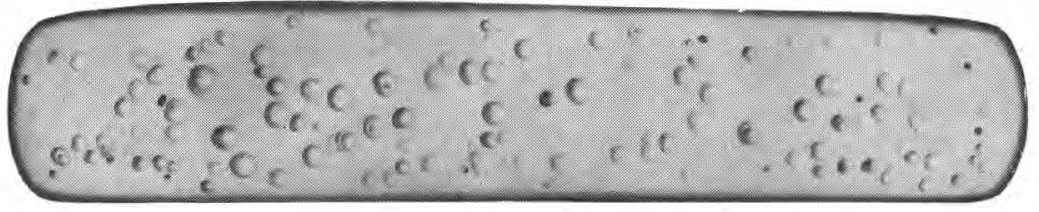

Fig. 1.

A $1233 \mathrm{E}$

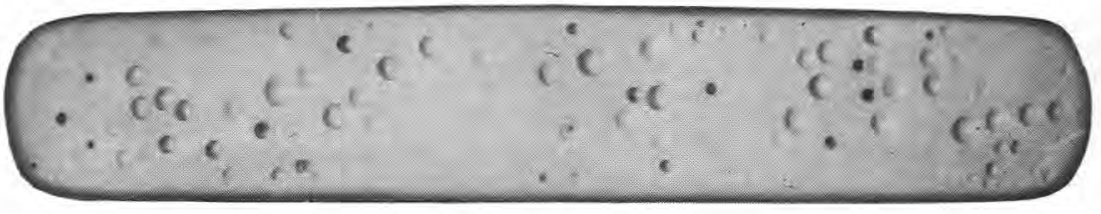

A $1232 \mathrm{~T}$

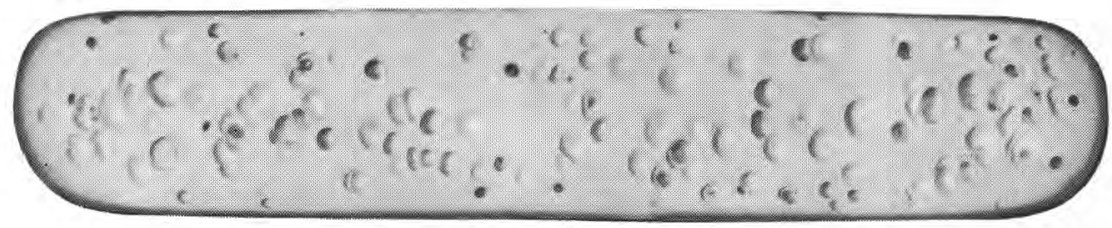

Fig. 2. 
Pour la fromagerie A la différence est très faible pour la première période ( 4 points) mais elle atteint 12 points pour les $2^{\mathrm{e}}$ et $3^{\mathrm{e}}$ périodes. Sa valeur moyenne pour les 3 périodes est de 9 points. Cette différence dans la notation correspond au fait que les fromages d'essai montraient généralement une meilleure ouverture, moins nombreuse et plus belle, que les fromages témoins. On peut voir à titre d'exemple, sur les figures 1 et 2 , deux paires de fromages de cette fromagerie (A 2913 essai et A 2912 témoin, A 1233 essai et A 1232 témoin) pour lesquels l'amélioration de l'ouverture grâce à l'utilisation de suspensions concentrées et congelées est nette.

Pour la fromagerie B, la différence moyenne entre fromages d'essai et fromages témoins est très faible ( 3 points), en faveur des fromages d'essai. Dans la première et la troisième périodes les fromages d'essai ont été mieux notés que les témoins (différences 5 et 10 points). Ces différences sont dues essentiellement aux meilleures notes d'ouverture attribuées aux fromages d'essai (fig. 3, B 8 essai et B 9 témoin). Par contre dans la deuxième période les fromages témoins ont été mieux notés que les fromages d'essai.

Pour la fromagerie C la différence est particulièrement nette pendant la première période où elle atteint 35 points. En plus de l'amélioration de l'ouverture (fig. 4, C 10 essai et C 12 témoin) on notait souvent un meilleur goût des fromages d'essai. On peut supposer que les levains traditionnels utilisés à ce moment-là par le fromager étaient responsables de la médiocrité de sa fabrication. Pendant la seconde période, dans la même fromagerie, les fromages témoins obtiennent les plus mauvaises notes de toute l'expérience $(62,2)$ et, si l'utilisation de levains actifs n'arrive pas à compenser totalement les mauvaises conditions de travail, elle provoque cependant une amélioration sensible (14 points) par la diminution des défauts d'ouverture et de goût (fig. 5, C 113 essai et C 213 témoin). Pour la troisième période l'écart est plus petit, mais encore net. Dans l'ensemble l'écart est de 17 points sur la moyenne des 3 périodes. Ces écarts de notation assez importants sont dûs à des différences de pâte, d'ouverture, mais surtout de goût. Les fromages témoins présentaient assez souvent des défauts de goût qui n'apparaissaient pas ou qui étaient beaucoup moins apparents sur les fromages d'essai.

Pour la fromagerie D, les différences entre essais et témoins sont également marquées pour les deux premières périodes pendant lesquelles elles atteignent 18 et 15 points. Pour la troisième période la différence est très faible ( 2 points) et en faveur des témoins. L'amélioration moyenne pour les 3 périodes atteint 10 points. Comme dans le cas de la fromagerie $\mathrm{C}$, les différences de notation concernaient surtout le goût des fromages. Cependant dans quelques cas, on trouvait aussi une ouverture nettement plus belle dans les fromages d'essai que dans les fromages témoins. C'est en parti- 
B $8 \mathrm{E}$

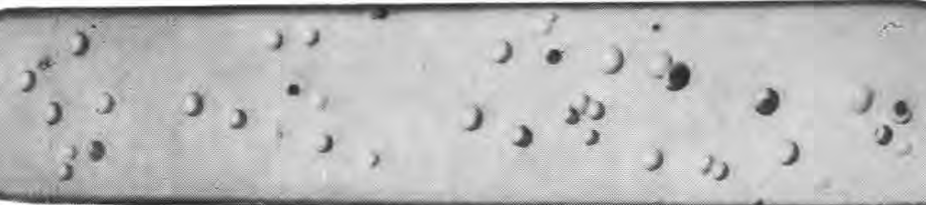

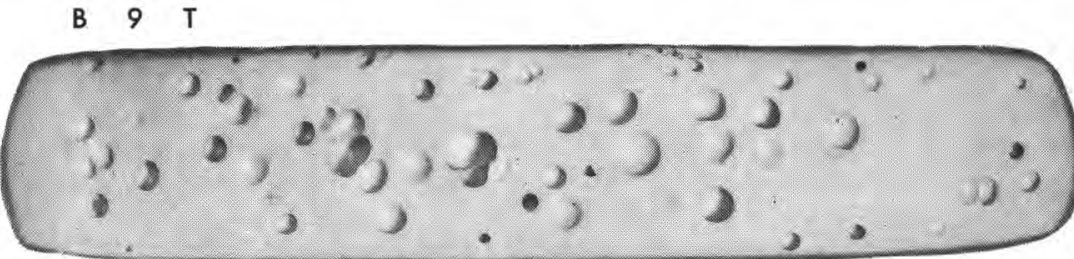

Fig. 3.

C $10 \mathrm{E}$

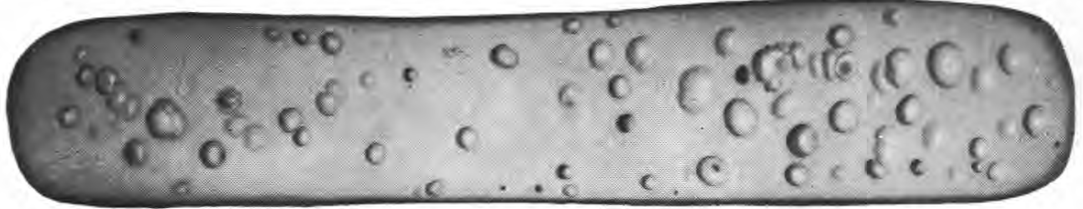

C $12 \mathrm{~T}$

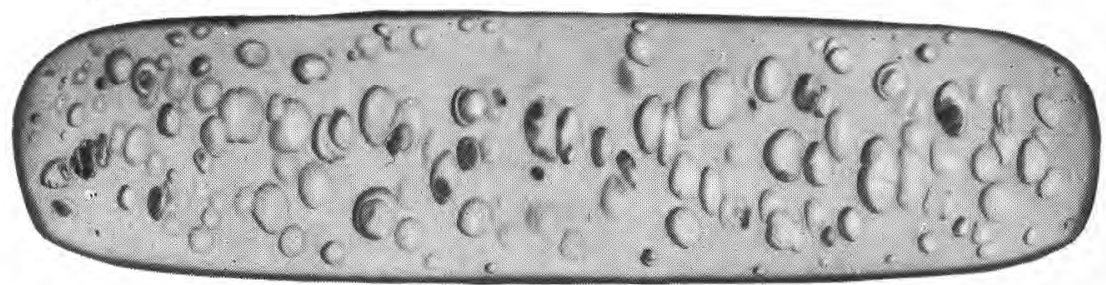

Fig. 4. 


\section{C $113 \mathrm{E}$}

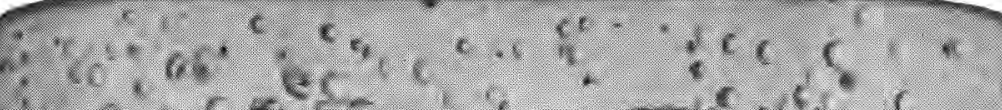

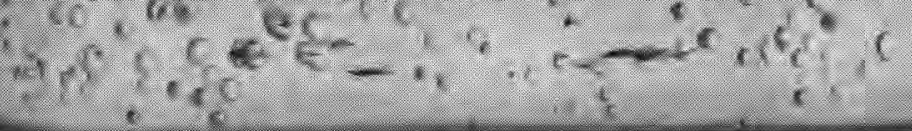

C $213 \mathrm{~T}$

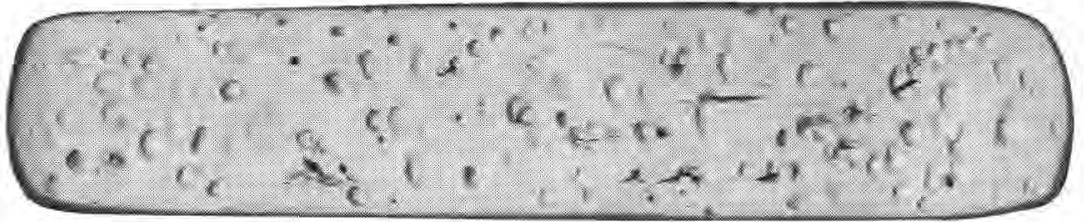

Fig. 5 .

D $8 E$

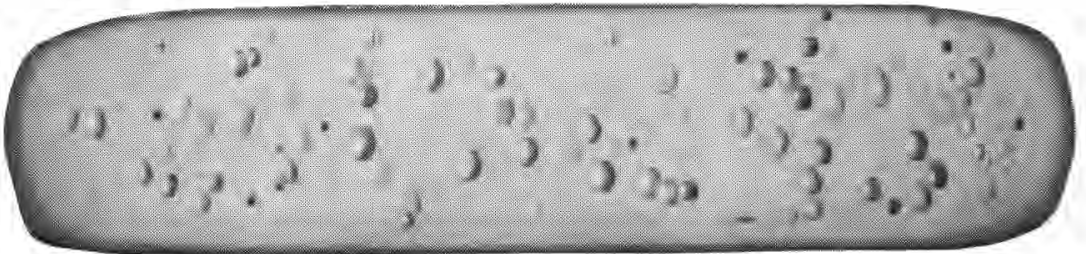

D $7 \mathrm{~T}$

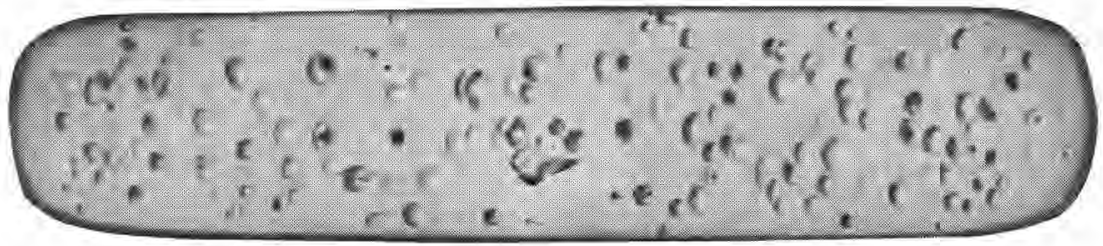

Fig. 6. 
culier le cas de la paire de fromages D 8 essai et D 7 témoin représentée sur la figure 6 , où l'on voit que l'utilisation de suspensions concentrées congelées a abouti à l'élimination de petits défauts d'ouverture ("becs de lainure", petite ouverture sous croûte).

\section{Discussion}

L'expérimentation que nous venons de décrire montre qu'il est tout à fait possible d'utiliser comme levain thermophile, dans la fabrication du Gruyère, des suspensions concentrées de streptocoques et de lactobacilles à la place des levains traditionnels. Ces suspensions concentrées peuvent se conserver plusieurs mois à l'état congelé $\left(-30^{\circ} \mathrm{C}\right)$ sans perte notable de leur activité.

Dans l'ensemble, l'utilisation de cette technique, dans les quatre fromageries du Jura où elle a été expérimentée, a permis souvent de constater une amélioration de la qualité des fromages. Cette amélioration était surtout nette dans le cas des fromageries dont les fabrications étaient déjà relativement satisfaisantes. On a alors observé une amélioration de l'ouverture et éventuellement du goût dans le cas des fromages fabriqués à l'aide des suspensions concentrées et congelées. Cette amélioration peut sans doute s'expliquer par le fait que l'emploi des suspensions bactériennes concentrées permet de régulariser l'ensemencement du lait : en apportant chaque jour un levain possédant toujours la même activité, on peut ainsi obtenir une acidification plus régulière du fromage sous presse.

Dans les conditions de l'expérience en fromagerie ce point revêtait certainement une importance particulière. En effet, en période d'hiver, les salles de pressage se refroidissent assez rapidement. Il est alors essentiel de disposer d'un levain toujours suffisamment actif pour provoquer l'égouttage du fromage avant que la température de la partie périphérique (talon) ne soit to mbée au-dessous de la zone favorable à la croissance des bactéries thermophiles.

Pour les très bonnes fromageries, l'avantage essentiel que le fromager peut trouver dans l'utilisation des levains concentrés est essentiellement d'ordre pratique, puisque la préparation des levains est ainsi supprimée.

Quant aux fromageries dont le niveau de qualité est médiocre, on ne peut espérer que l'emploi de levains concentrés puisse permettre à ces fromageries d'obtenir régulièrement des fromages de bonne qualité. Les défauts de fabrication peuvent provenir aussi bien du lait et/ou de la technique de fabrication que du levain lui-même. Cependant il est certain que l'emploi de suspensions concentrées devrait permettre dans beaucoup de cas d'éliminer une partie des défauts de fabrication.

(Reçu pour publication en mars 1968.) 


\section{Remerciements}

Nous remercions MM. les présidents et fromagers des fruitières de Foncine-le-Haut, Montrond, Ney et Salins (Jura) pour l'accueil qu'ils nous ont réservé et l'aide qu'ils nous ont apportée dans la réalisation de ces essais.

Nous tenons également à remercier les Etablissements Brun, affineur à Poligny, Grillot, affineur à Ornans, Rivoire et Jacquemin, affineur à Montmorot, pour le concours précieux que nous avons toujours trouvé auprès d'eux.

\section{Résumé}

Sept fabrications de Gruyère ont été effectuées à la laiterie expérimentale de Jouy-en-Josas, en utilisant comparativement des levains préparés sur lait stérilisé et des suspensions concentrées congelées de Str. thermophilus et de $L$. helveticus. L'acidification $d u$ fromage sous presse était plus rapide et plus régulière pour les fromages d'essai fabriqués à l'aide des suspensions bactériennes concentrées qu'avec les fromages témoins fabriqués à l'aide des levains sur lait. L'extrait sec après 24 heures de pressage et le rendement en fromage frais étaient semblables pour les fromages d'essai et pour les fromages témoins.

Les mêmes suspensions concentrées congelées ont été utilisées pendant quatre mois dans quatre fromageries du Jura. Les fromageries fabriquaient chaque jour simultanément, pendant les périodes d'essai, un fromage d'essai à l'aide des suspensions concentrées congelées, et un fromage témoin en employant un levain traditionel (" présure aux caillettes"). Après affinage, les fromages étaient coupés et notés par un jury d'experts. En moyenne les fromages d'essai ont été mieux notés, que les fromages témoins. Cette différence de notation correspondait en général à une "ouverture " plus régulière et à un goût meilleur des fromages d'essai.

Ces essais montrent qu'il est tout à fait possible d'utiliser comme levain thermophile, dans la fabrication du Gruyère, des suspensions concentrées congelées de streptocoques et de lactobacilles à la place des levains traditionnels.

\section{Summiary}

\section{The use of concentrated frozen suspensions of thermophilic lactic acid bacteria in making Gruyère-cheese.}

Seven pairs of Gruyère-cheese were made at the Expérimental Dairy, Jouy-en-Josas, using for comparison either starters prepared with sterilized milk or concentrated frozen suspensions of Str. 
thermophilus and $L$. helveticus. The acid production in the cheeses under the press was more rapid and more uniform for the experimental cheeses made with the concentrated suspensions than for the control cheeses made with the milk starters.

Similar concentrated frozen suspensions have been used for 4 months in 4 cheese factories in the Jura. The factories made each day one experimental cheese with the concentrated frozen suspensions and one control cheese using traditional starters (calf stomach preparations). After ripening, the cheeses were cut, and marks were given by a panel of experts. On an average the experimental cheeses were given higher marks than the control cheeses. This difference in the marks was mainly due to a more uniform distribution of the holes and to a better taste in the experimental cheeses.

This experiment shows that it is quite feasible to use, concentrated frozen suspensions of thermophilic streptococci and lactobacilli in place of the traditional starters in making Gruyère-cheese.

\section{BIBLIOGRAPHIE}

[1] Accolas (J.-P.) et Auclair (J.), 1967. Conservation à l'état congelé de suspensions de bactéries lactiques concentrées sous faible volume. I. Bactéries lactiques mésophiles. Le Lait, 465, 253-260.

[2] Valles (E.) et Mocquot (G.), 1968. Préparation de suspension concentrées congelées de bactéries lactiques thermophiles destinées à la fromagerie. Le Lait (en préparation). 